\title{
Normal Incidence of Sound Transmission Loss from Perforated Plates with Micro and Macro Size Holes
}

\author{
A. Putra ${ }^{1}$ and A. Y. Ismail ${ }^{2}$ \\ ${ }^{1}$ Centre for Advanced Research on Energy, Universiti Teknikal Malaysia Melaka, 76100 Hang Tuah Jaya, Melaka, Malaysia \\ ${ }^{2}$ Faculty of Engineering Technology, Universiti Teknikal Malaysia Melaka, 76100 Hang Tuah Jaya, Melaka, Malaysia \\ Correspondence should be addressed to A. Putra; azma.putra@utem.edu.my
}

Received 7 February 2014; Revised 26 March 2014; Accepted 28 March 2014; Published 17 April 2014

Academic Editor: Rama B. Bhat

Copyright (C) 2014 A. Putra and A. Y. Ismail. This is an open access article distributed under the Creative Commons Attribution License, which permits unrestricted use, distribution, and reproduction in any medium, provided the original work is properly cited.

This paper studies the sound transmission loss of perforated panels and investigates the effect of the hole diameter on the sound insulation performance under normal incidence of acoustic loading. The hole diameters are distinguished into micro (submillimeter) and macro (millimeter) sizes. In general, the transmission loss reduces as the perforation ratio is increased. However, by retaining the perforation ratio, it is found that the transmission loss increases as the hole diameter is reduced for a perforate with micro holes due to the effect of resistive part in the hole impedance, which is contrary to the results for those with the macro holes. Both show similar trend at high frequency where the fluid behavior inside the hole is inertial. Simple analytical formulae for engineering purpose are provided. Validation of the models with measurement data also gives good agreement.

\section{Introduction}

Perforated panels are commonly found in acoustics and noise control applications, for example, as a facing for porous material or as a structure in machinery. For the former, the perforate acts more as the protective layer for the porous acoustic material but at the same time influences the surface impedance affecting the sound absorption. For the latter, introduction of holes reduces the surface volume velocity of a vibrating structure which then reduces the structural noise radiation. For both practices, the perforate is typically constructed with hole size which is obvious for one to observe (usually $>1 \mathrm{~mm}$ ). A perforated plate with submillimeter holes becomes well known recently as a non-fibrous sound absorber. Backed by an air layer in front of a rigid surface, this type of perforate behaves like a Helmholtz resonator which optimally absorbs sound energy at its resonant frequency. For optimum absorption, this microperforated panel (MPP) should have hole size ranging between 0.05 and $1 \mathrm{~mm}$ and with perforation ratio of $0.5 \%-1.5 \%[1]$.

Several works have been published to discuss the performance of the perforates in terms of their sound absorption and sound radiation. For examples, Lee et al. [2] investigated the effect of modal vibration on a MPP which is found to widen the frequency bandwidth of the absorption. Pfretzschner et al. [3] show that a MPP can be coupled with a thick perforated plate to increase structural strength of the absorber and at the same time also increases the absorption frequency range into two or three octave bands. A suspended MPP system without rigid backing is also found to have good sound absorption in application [4]. Sakagami et al. [5] also present that a double-leaf MPP absorber consisting of two MPPs without rigid backing improves the sound absorption at low frequencies. Toyoda et al. [6] proposed a perforated system backed with honeycomb structure both as an absorber and as a low-radiation panel. The capability of a perforate to reduce sound radiation has also been modelled by Putra and Thompson [7] which shows that effective reduction can be obtained for a perforated panel with many small holes rather than that with few large holes (with the same perforation ratio).

Some studies have also been carried out to investigate the performance of the perforate system as sound insulator. Chen [8] modelled the transmission loss of a rigid perforated 
screen using two-dimensional plane wave theory. The results show reasonable agreement with measurement. Takahashi and Tanaka [9] used the Helmholtz integral technique to numerically calculate the sound pressure at both sides of an infinite perforated plate. The sound transmission loss obtained here considers the flexural vibration of the panel. The results show that the transmission loss reduces as the perforation ratio increases. The use of MPP as noise barrier was highlighted by Asdrubali and Pispola [10] by designing a door consisting of three layers of MPPs. Toyoda and Takahashi [11] highlighted their similarly previous perforate model [6] in terms of the sound insulation performance, but now of a MPP backed with a honeycomb board. By subdividing the air cavity in the air gap between the MPP and the back wall, it is shown that the transmission loss at mid frequencies can be improved. Most recently, Mu et al. [12] present the sound insulation performance of a multilayer partition with a MPP (at the outer layer) to prevent the phenomenon of mass-air mass resonance. Similar study was also carried out by Putra et al. [13], but for the case of MPP located between two solid plates.

In this paper, analytical model using the plane wave theory for sound transmission loss of a perforate as a single partition is proposed. This study is however limited for the case of normal incidence of acoustic excitation, where in practice, the sound usually comes from various angles of incidence. This paper therefore emphasizes the discussion to be more on the effect of hole diameter on the transmission loss in which the similar phenomena might be found for the case of oblique or diffuse field incidence. The term "micro" denotes the submillimetric diameter and "macro" for the millimetric size. Simple analytical formulae are also proposed at particular frequency range which can be used as practical guidance in noise control. The models are also validated with experimental data.

\section{Governing Equations}

2.1. The Average Surface Velocity. Figure 1 shows the diagram of a perforated panel subjected under normal incidence of acoustic loading. The air particles penetrate the holes and also excite the remaining solid surface of the panel and set it into motion. The average particle velocity over the plate surface $\bar{v}$ is the combination of the particle velocity due to the motion of the panel $v_{p}$ and the particle velocity inside the hole $v_{h}$ given by [9]

$$
\bar{v}=v_{p}(1-\sigma)+\sigma v_{h}
$$

where $\sigma$ is the perforation ratio. Inside the holes, the air moves like a moving piston due to its inertial property while at the same time it interacts with the inner surface of the holes creating friction force due to its viscous effect. Both mechanisms can be represented in terms of the hole impedance $Z_{h}$ which has been proposed by Maa [1]:

$$
Z_{h}=Z_{h, R}+Z_{h, I}
$$

with

$$
\begin{aligned}
& Z_{h, R}=\frac{32 v_{a} t}{d_{o}^{2}}\left[\left(1+\frac{X_{o}^{2}}{32}\right)^{1 / 2}+\left(\frac{\sqrt{2} X_{o}}{8}\right) \frac{d_{o}}{t}\right], \\
& Z_{h, I}=j \rho \omega t\left[1+\left(9+\frac{X_{o}^{2}}{2}\right)^{-1 / 2}+\left(\frac{8}{3 \pi}\right) \frac{d_{o}}{t}\right],
\end{aligned}
$$

where $X_{o}=\left(d_{o} / 2\right)\left(\omega \rho / v_{a}\right)^{1 / 2}, d_{o}$ is the hole diameter, $\omega$ is the angular frequency, $\rho$ is the air density, $t$ is the plate thickness, and $v_{a}$ is the viscosity of the air which is $1.8 \times$ $10^{-5} \mathrm{Ns} / \mathrm{m}^{2}$. The real part of the impedance $Z_{h, R}$ represents the viscous effect and the imaginary part $Z_{h, I}$ represents the inertia of the air inside the holes. Figure 2 presents the magnitude of the hole impedance against the hole diameter. It can be seen that for micro size holes below $1 \mathrm{~mm}$, the resistive part dominates the hole impedance particularly at very low frequency where here, the motion of the fluid inside the hole is predominantly controlled by the friction between the air and the inner surface of the hole. This real part, however, reduces rapidly as the diameter is increased which then results in the domination of the reactive part in the impedance. The real part also becomes lower than the imaginary part as the frequency increases. The net pressure on the surface of the panel can therefore be given by

$$
Z_{h, R}\left(v_{h}-v_{p}\right)+Z_{h, I} v_{h}=\Delta p,
$$

where the first term on the left hand side is the force per unit area due to the friction which is proportional to the relative motion between the fluid and the plate and the second term is due to the inertia of the air inside the hole. Equation (5) can also be rearranged as

$$
v_{h}-v_{p}=\frac{\Delta p}{Z_{h}}-\frac{Z_{h, I}}{Z_{h}} v_{p} .
$$

By substituting this into (1), the average surface velocity can also be expressed as the function of the net pressure given by

$$
\bar{v}=\gamma v_{p}+\frac{\Delta p}{z_{h}},
$$

where $z_{h}=Z_{h} / \sigma$ is the uniform specific acoustic impedance assuming distribution of holes across the plate surface and $\gamma=1-\left(Z_{h, I} / z_{h}\right)$ is a complex nondimensional term.

2.2. The Normal Incident Transmission Loss. Consider a solid panel which is uniform, unbounded, and nonflexible having mass per unit area $m$ supported by viscous dampers $r$ and elastic suspensions $s$ per unit area as shown in Figure 1. An idealised normal incidence of sound is assumed to impinge the panel with frequency $\omega$. The total sound pressure at the left hand side of the panel is hence

$$
p^{-}(x)=P_{i} e^{-j k x}+P_{r} e^{j k x},
$$




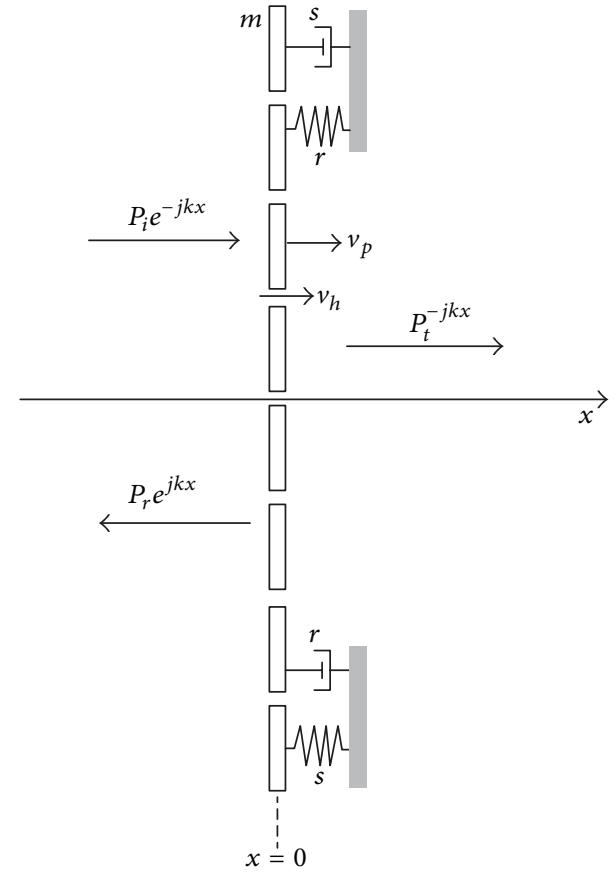

FIGURE 1: A schematic diagram of a perforated panel excited by normal incidence of sound wave.

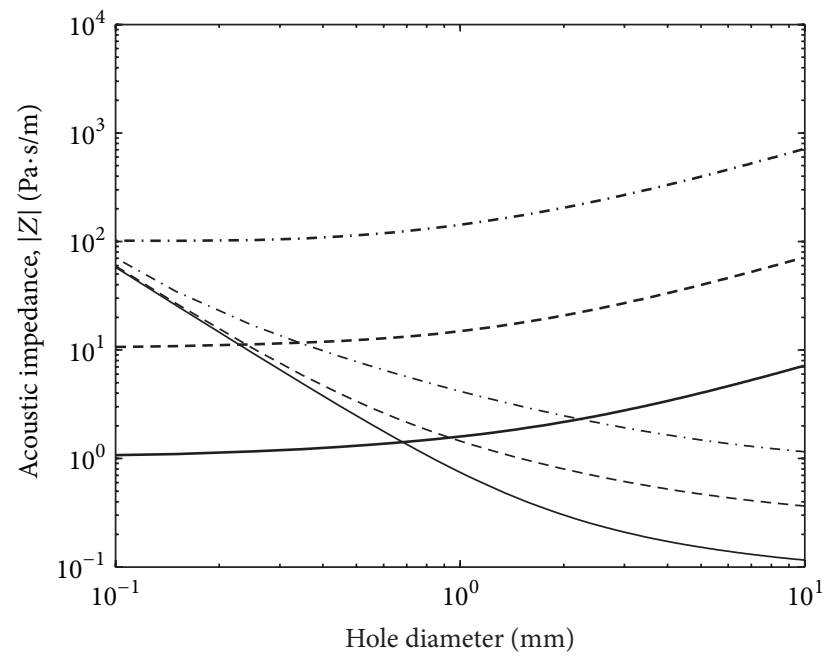

FIgURE 2: The magnitude of the real (thin lines) and imaginary (thick lines) parts of the acoustic impedance of a circular hole in a $1 \mathrm{~mm}$ thick plate $(-100 \mathrm{~Hz},--1 \mathrm{kHz}$, and $-\cdot-10 \mathrm{kHz})$.

where $P_{i}$ and $P_{r}$ denote the complex amplitude of the incident pressure and reflected pressure, respectively, $k=\omega / c$ for $k$ represents the acoustic wavenumber, and $c$ is the sound speed in the air. The first term at the right hand side of (8) is the incident sound pressure and the second term is the reflected sound pressure. For the rest of the equations, time dependence $e^{j \omega t}$ is implicitly assumed.

The relation between the average surface velocity (at $x=$ 0 ) and the sound pressure exciting the panel can be obtained by using Euler equation $\bar{v}=-1 / j \rho \omega(\mathrm{d} p / \mathrm{d} x)$ which from (8) gives

$$
P_{i}-P_{r}=z_{f} \bar{v},
$$

where $z_{f}=\rho c$ is the impedance of air. At the panel surface, by substituting $P_{r}$ from (9) into (8) yields

$$
p^{-}(x=0)=2 P_{i}-z_{f} \bar{v} .
$$

The radiated pressure caused by the panel acceleration in the positive $x$ direction is expressed as

$$
p^{+}(x)=P_{t} e^{-j k x},
$$

where $P_{t}$ is the complex amplitude of the transmitted pressure and again by using the Euler equation at $x=0$ gives

$$
P_{t}=z_{f} \bar{v}
$$

By substituting (12) into (10) gives the total pressure on the left hand side of the surface of the panel:

$$
p^{-}(x=0)=2 P_{i}-P_{t} .
$$

The total pressure on the right side of panel $p^{+}$has been represented by the radiated field in (12) which is equivalent to the transmitted pressure. The pressure difference across the surface of the panel is

$$
\Delta p=p^{-}-p^{+}=2\left(P_{i}-P_{t}\right) .
$$

2.2.1. Micro Size Diameter. As only normal incident of sound is assumed to be exciting the panel, the system can be considered as a single degree of freedom system (where the whole surface of the panel moves in phase in $x$ direction). It is the mass of the panel which is important in this case. The equation of motion of the system with submillimetric holes is expressed as

$$
z_{p} v_{p}=\Delta p(1-\sigma)+Z_{h, R}\left(v_{h}-v_{p}\right) \sigma,
$$

where $v_{p}$ is the panel velocity. The first term on the right hand side of (15) is the force acting to the solid part of the panel due to the sound pressure difference and the second term is the acting friction force inside the holes. The panel in vacuo mechanical impedance is given by

$$
z_{p}=j \omega m-\frac{j s}{\omega}+r=j \omega m\left(1-\frac{j \eta \omega_{n}}{\omega}\right)-\frac{j s}{\omega},
$$

where the mechanical damping per unit area has been replaced by $r=\omega_{n} \eta m$ with $\omega_{n}=\sqrt{s / m}$ being the undamped natural frequency and $\eta$ is the mechanical loss factor. By substituting (6) and (14) into (15) yields the panel velocity which is expressed as

$$
v_{p}=\frac{2\left(P_{i}-P_{t}\right) \delta}{z_{q}},
$$


with

$$
z_{q}=z_{p}+\frac{Z_{h, R} Z_{h, I}}{z_{h}}
$$

where $\delta=1-\sigma+\left(Z_{h, R} / z_{h}\right)$ as in (7) is also a complex nondimensional term. Equation (17) is then substituted back into (7) to obtain the ratio of the transmitted pressure amplitude to that of the incident pressure:

$$
\frac{P_{t}}{P_{i}}=\frac{2 z_{f}}{z_{r}+2 z_{f}}
$$

where

$$
z_{r}=\frac{z_{h}}{1+\gamma \delta z_{h} / z_{q}} .
$$

As for a plane wave, the sound power is proportional to the sound intensity $|p|^{2} / \rho c$. The power transmission coefficient $\tau$ is therefore given by

$$
\tau=\left|\frac{P_{t}}{P_{i}}\right|^{2}=\left|\frac{2 z_{f}}{z_{r}+2 z_{f}}\right|^{2} .
$$

2.2.2. Macro Size Diameter. For a partition introduced with millimetric size holes $\left(d_{o} \geq 1 \mathrm{~mm}\right)$, the behaviour of the air inside the holes becomes purely inertial. The reactive part in (4) for macro size hole can be expressed as [7]

$$
Z_{h, I}=j h z_{f},
$$

where

$$
h=k\left[t+\left(\frac{8}{3 \pi}\right) d_{o}\right]
$$

is the nondimensional acoustic reactance. Here the second term inside the bracket in (4) has been ignored as $(9+$ $\left.X_{o}^{2} / 2\right)^{-1 / 2} \ll 1$. The second term in (23), that is, $(8 / 3 \pi) d_{o}$, corresponds to the end correction at both ends of the hole, which is proportional to an added mass in the proximity of a moving piston [14]. This term will dominate for $d_{o} \gg t$. The equation of motion can therefore be expressed as

$$
z_{p} v_{p}=\Delta p(1-\sigma)
$$

with $Z_{h, I} \gg Z_{h, R}$; hence, $Z_{h} \approx Z_{h, I}$ and the complex nondimensional terms become $\gamma=\delta=1-\sigma$. The mean particle velocity in (7) is therefore

$$
\bar{v}=v_{p}(1-\sigma)+\frac{\Delta p}{z_{h, I}},
$$

where $z_{h, I}=Z_{h, I} / \sigma$. The transmission coefficient in (21) can now be written as

$$
\tau=\left|\frac{2 z_{f}}{z_{s}+2 z_{f}}\right|^{2}
$$

where

$$
z_{s}=\frac{z_{h, I}}{1+(1-\sigma)^{2} z_{h, I} / z_{p}} .
$$

From (21) and (26), the transmission coefficient for a solid panel can be obtained by setting $\gamma=1, \sigma=0, \delta=0$, and $z_{q}=z_{r}=z_{s}=z_{p}$ which gives

$$
\tau=\left|\frac{2 z_{f}}{z_{p}+2 z_{f}}\right|^{2} .
$$

Finally the transmission loss in decibel $(\mathrm{dB})$ unit can be calculated by

$$
\mathrm{TL}=10 \times \log _{10}\left(\frac{1}{\tau}\right)
$$

\section{Results}

3.1. Stiffness Controlled Region. Figure 3 shows the analytical result of sound transmission loss (TL) of perforated panels having micro and macro size holes. The calculation is conducted for an aluminium plate with density $2700 \mathrm{~kg} / \mathrm{m}^{3}$ and thickness $1 \mathrm{~mm}$. The stiffness per unit area of the mounting is set to $300 \mathrm{kN} / \mathrm{m}^{3}$ in order to observe its effect on the results. This can be seen for the solid plate results at very low frequencies where the STL decreases with frequency converging to zero value towards the undamped natural frequency $\omega_{n}$ at around $40 \mathrm{~Hz}$.

For the microperforated panels in Figures 3(a) and 3(b), the TL below the natural frequency particularly for $d_{0}=$ $0.1 \mathrm{~mm}$ is the same as that of the solid panel. This is due to a large resistive force which restricts the motion of the air inside the holes. At frequency well below the natural frequency that is, $\omega \ll \omega_{0}$, and where the loss factor $\eta$ is typically much less than unity, from (16) and (18) this yields $z_{q}=z_{p} \approx-j s / \omega$; the mechanical impedance is primarily determined by the elastic stiffness. As the resistive part dominates the hole impedance $Z_{h, R} \gg Z_{h, I}$, from (20) and (21), the transmission loss in (29) can then be written as

$$
\mathrm{TL} \approx 10 \log _{10}\left[\frac{z_{h, R}^{2}}{1+\left(\omega z_{h, R} / s\right)^{2}}\right]-58,
$$

where $z_{h, R}=Z_{h, R} / \sigma$ and the impedance of the air is $z_{f}=\rho c=$ $412 \mathrm{~kg} \mathrm{~m}^{-2} \mathrm{~s}^{-1}$. When the resistive impedance is so large that it exceeds the mechanical stiffness impedance, $z_{h, R} \gg s / \omega$, (30) reduces to [15]:

$$
\mathrm{TL} \approx 20 \log _{10}(s)-20 \log _{10}(\omega)-58 .
$$

It can be seen that the TL is controlled by the stiffness of the mounting and insensitive to the presence of holes in the panel. The perforated panel hence acts like a solid panel. The second term indicates that the TL rolls off with frequency by $20 \mathrm{~dB} /$ decade (see again the result for solid plate or $d_{o}=$ $0.1 \mathrm{~mm}$ ). 


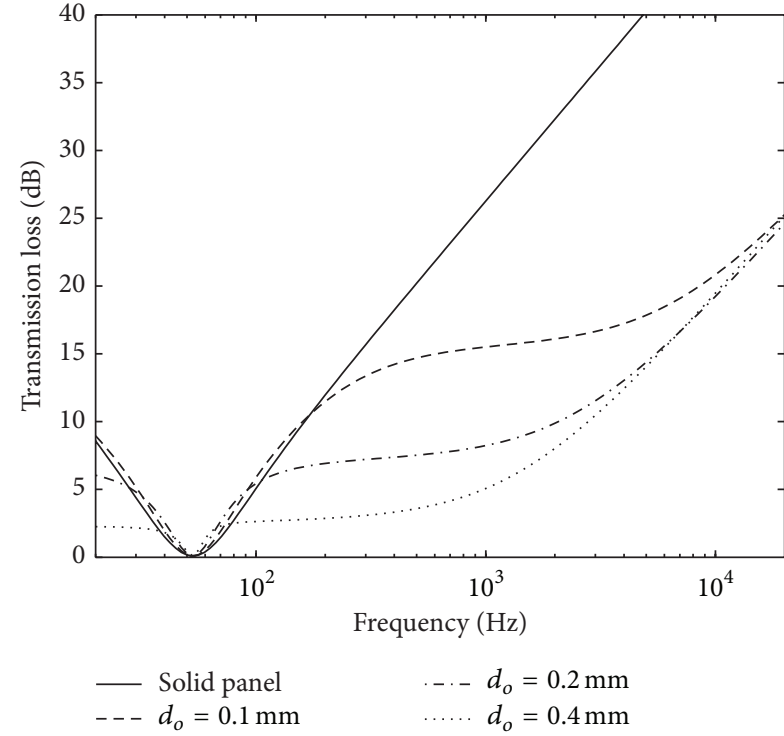

(a)

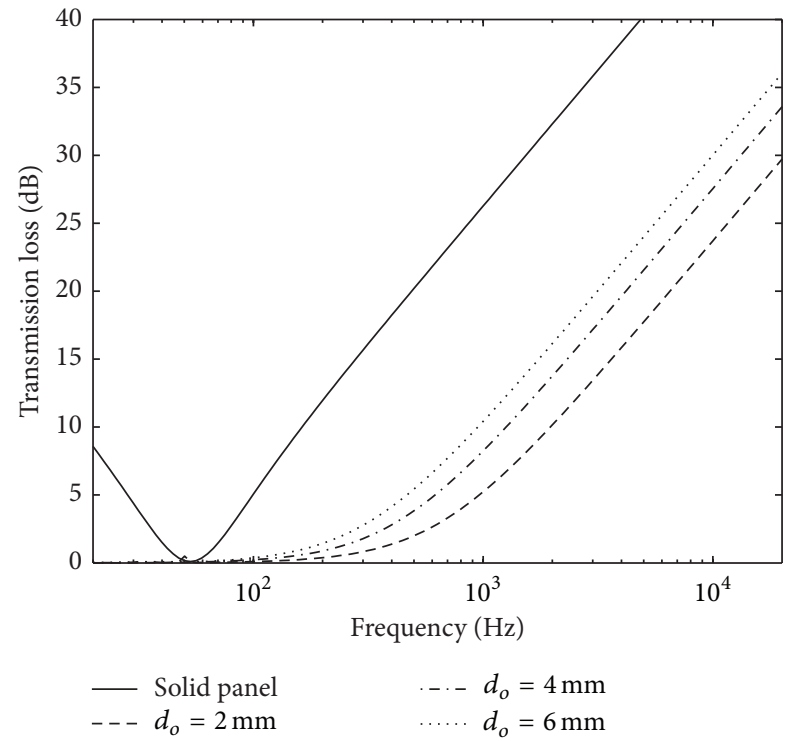

(c)

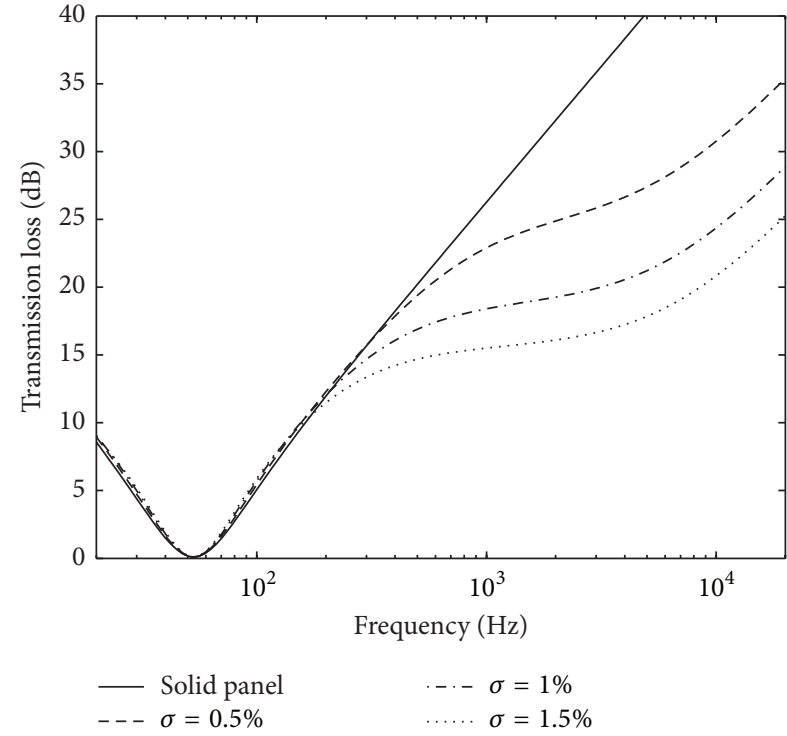

(b)

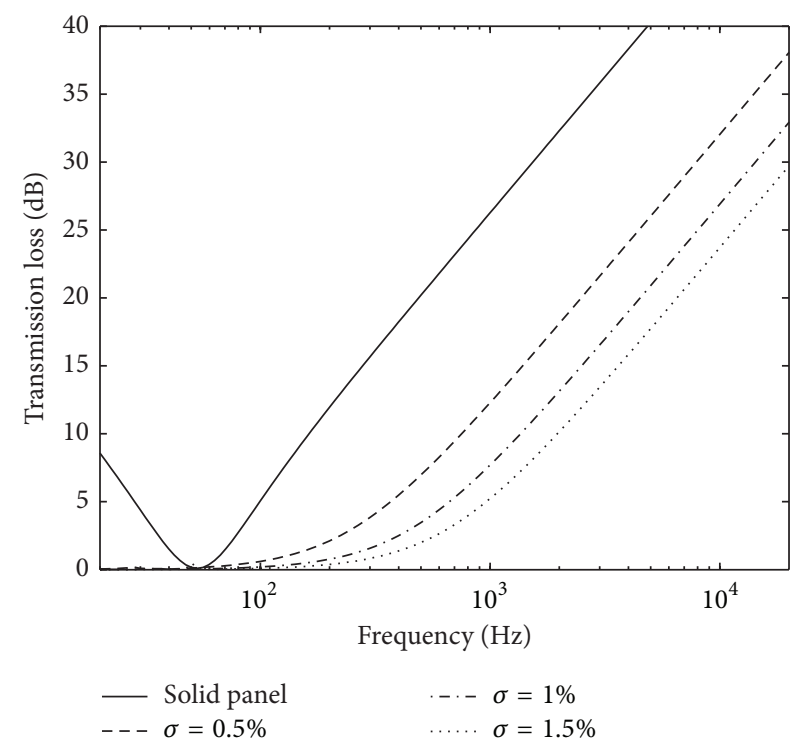

(d)

FIGURE 3: Transmission loss of aluminium perforated plates having thickness $t=1 \mathrm{~mm}$ with (a)-(b) micro size holes and (c)-(d) medium size holes subjected to normal incidence of sound: (a) $\sigma=1.5 \%$, (b) $d_{o}=0.1 \mathrm{~mm}$, (c) $\sigma=1.5 \%$, and (d) $d_{o}=2 \mathrm{~mm}$.

The perforation, however, will affect the TL when the resistive force in the holes reduces as the hole diameter increases that it becomes smaller than the mechanical impedance, $z_{h, R} \ll s / \omega$. Equation (30) can then be expressed as

$$
\mathrm{TL} \approx 20 \log _{10}\left(z_{h, R}\right)-58
$$

The TL now depends on the real part of the hole impedance. As the real part reduces with the increasing hole diameter, the TL also reduces as seen for $d_{o}=2 \mathrm{~mm}$ and $d_{o}=4 \mathrm{~mm}$ below $40 \mathrm{~Hz}$.
Figures 3(c) and 3(d) plot the results when the hole diameter is increased to the macro size $\left(d_{o}>1 \mathrm{~mm}\right)$. The hole impedance is now dominated by the reactive part, $Z_{h, R} \ll$ $Z_{h, I}$. As this imaginary part of impedance is much smaller than the mechanical impedance at low frequency, $z_{h, I}<j s / \omega$, then from (27), $z_{s} \approx z_{h, I}$. The transmission loss is therefore

$$
\mathrm{TL} \approx 10 \log _{10}\left(1+\left|\frac{z_{h, I}}{2 z_{f}}\right|^{2}\right) .
$$



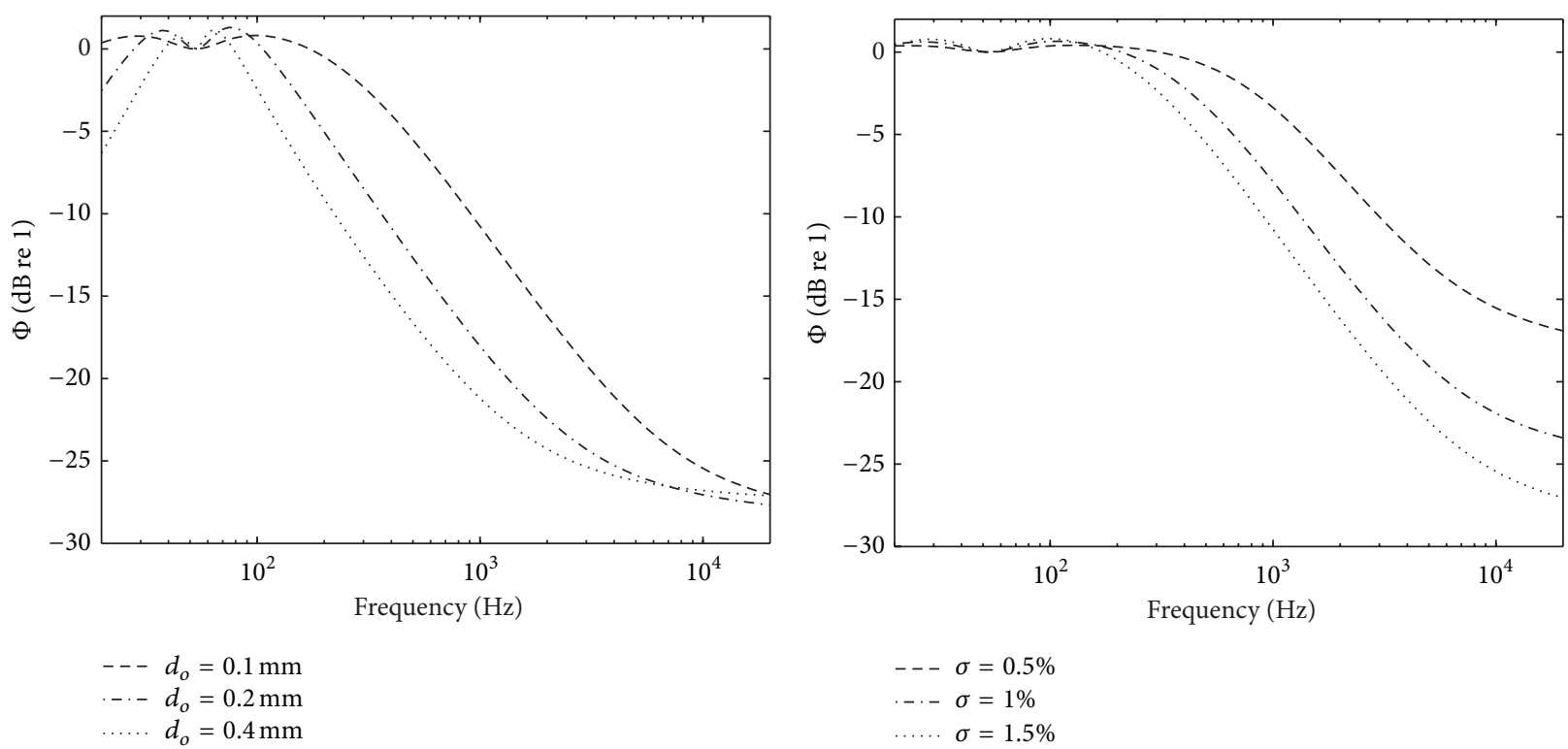

(a)

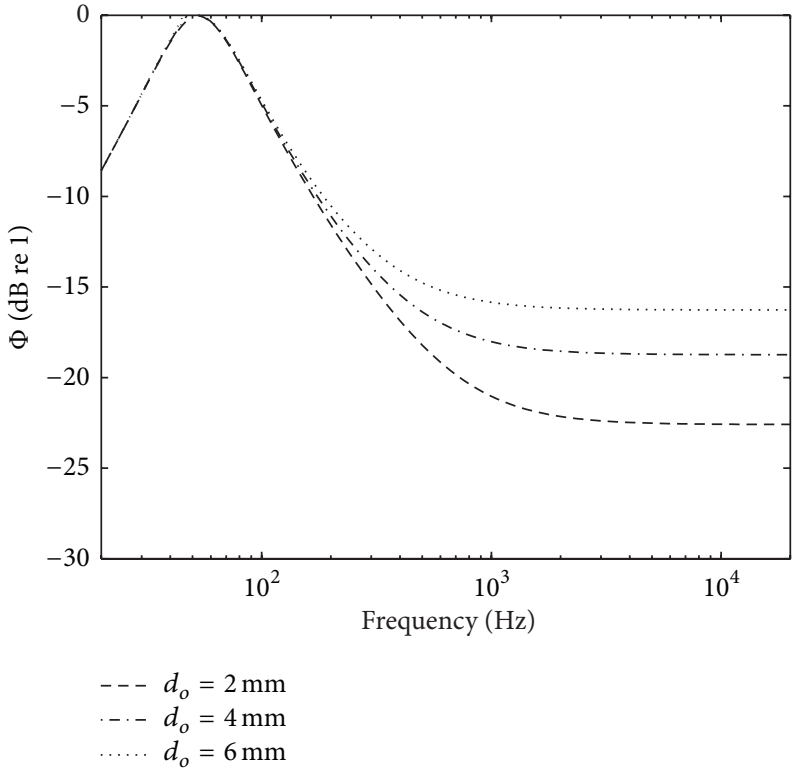

(c)

(b)

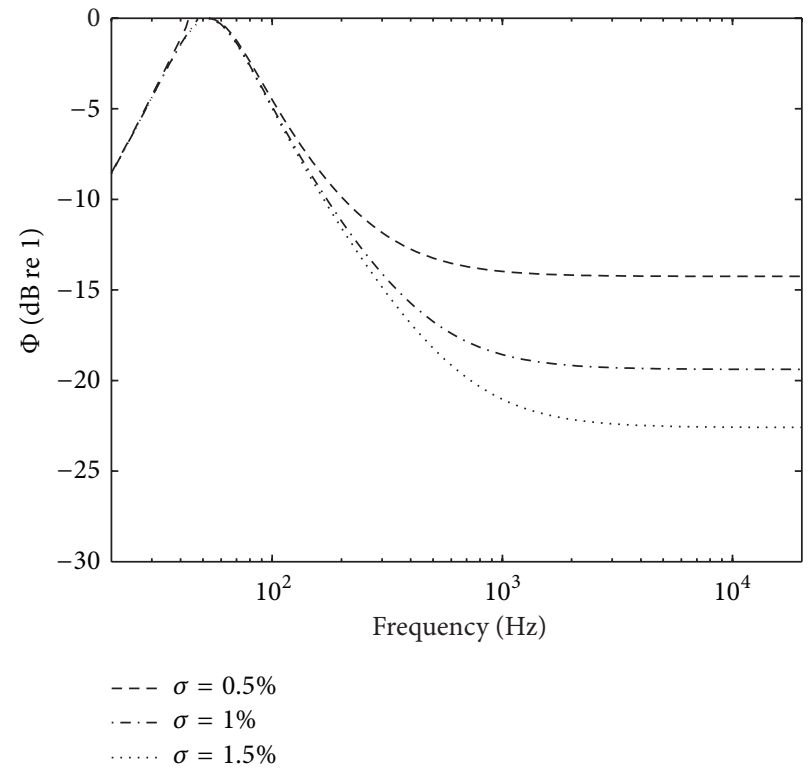

(d)

FIGURE 4: The effect of perforation on the transmission loss of aluminium perforated plates having thickness $t=1 \mathrm{~mm}$ with (a)-(b) micro size holes and (c)-(d) medium size holes subjected to normal incidence of sound: (a) $\sigma=1.5 \%$, (b) $d_{o}=0.1 \mathrm{~mm}$, (c) $\sigma=1.5 \%$, and (d) $d_{o}=2 \mathrm{~mm}$.

At low frequencies, particularly for a thin plate, the impedance of the air is much larger than the reactive part of the hole impedance, $z_{f} \gg z_{h, I}$. The TL in (33) therefore approaches zero as shown in Figures 3(c) and 3(d). With macro size holes, the sound energy can effectively transmit through the partition at very low frequency.

3.2. Mass Controlled Region. At the frequency well above the natural frequency $\omega \gg \omega_{n}$, from (18), $z_{q}=z_{p} \approx j \omega m$. Substituting this into (20) and after mathematical manipulation, the sound transmission loss for the microperforated panel is given by

$$
\begin{aligned}
\mathrm{STL} \approx & 20 \log _{10}(\omega m)+10 \log _{10}\left(\left[z_{h, R}+2 z_{f}\right]^{2}+\left|z_{h, I}\right|^{2}\right) \\
& -10 \log _{10}\left(z_{h, R}^{2}+\left[\omega m-\left|z_{h, I}\right|\right]^{2}\right)-58 .
\end{aligned}
$$

Equation (34) indicates that apart from the mass of the panel, the TL is controlled by both the resistive and 


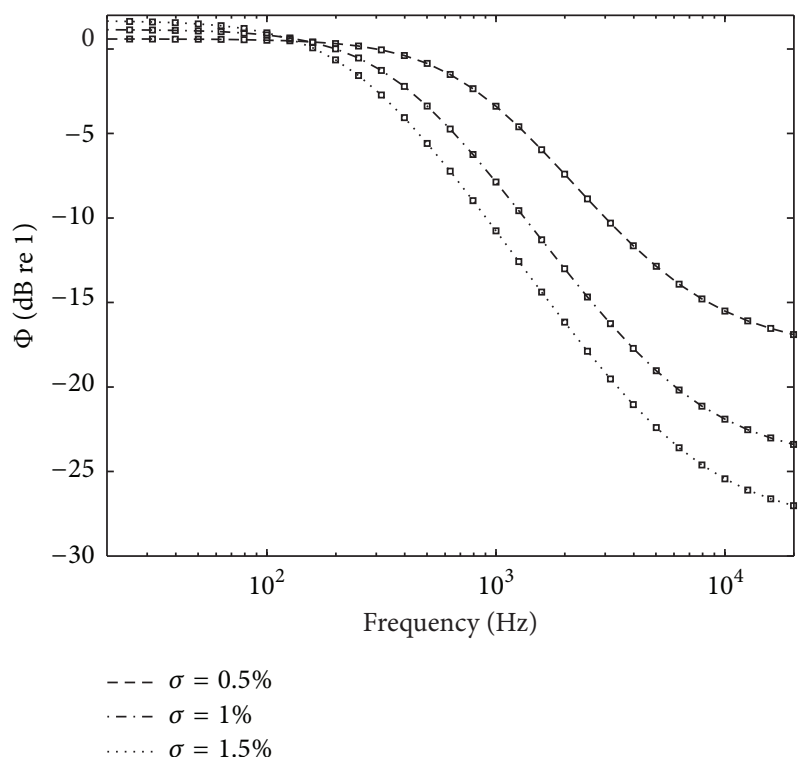

FIGURE 5: The effect of perforation for plates with micro size holes; $d_{o}=0.1 \mathrm{~mm}$ (marked line: approximated $\Phi$ at mass controlled region from (38)).

the imaginary parts of the hole impedance. At frequency just above the natural frequency, the real part still affects the TL which in Figure 3(a) is shown by almost constant TL below $1 \mathrm{kHz}$. At this frequency range, reduction of TL can be observed with increasing hole diameter due to the reduction of the friction force. Above $1 \mathrm{kHz}$, the TL can be seen to increase rapidly with frequency. This is where the reactive part is dominant over the resistive part. As from Figure 2, the imaginary part is nearly constant for diameter of hole below $d_{o}=1 \mathrm{~mm}$ and therefore as observed in Figure 3(a), at high frequency above $1 \mathrm{kHz}$ the TL is less sensitive to the change of hole diameter.

For plates with macro size holes in Figures 3(c) and $3(\mathrm{~d})$, the TL increases by $20 \mathrm{~dB} /$ decade for almost the entire frequency range above the natural frequency. For this case where the imaginary part is responsible for the TL, using (23) and (26) and for $z_{p} \approx j \omega m$, the TL is given by

$$
\begin{aligned}
\mathrm{TL} \approx & 20 \log _{10}(\omega m)+10 \log _{10}\left(1+\frac{4 \sigma^{2}}{h^{2}}\right) \\
& -20 \log _{10}\left(1+\frac{\omega m \sigma}{h z_{f}}\right)-58 .
\end{aligned}
$$

It is interesting to note that for this millimetric holes with fixed perforation ratio as shown in Figure 3(c), the TL reduces as the hole diameter becomes smaller. In other words, in the absence of friction force inside the holes, sound energy transmits more effectively in a plate with many smaller holes rather than with few larger holes. However, for plate with millimetric holes as in Figure 3(a), this shows a contrary finding where the TL reduces as the hole size increases with fixed perforation ratio.

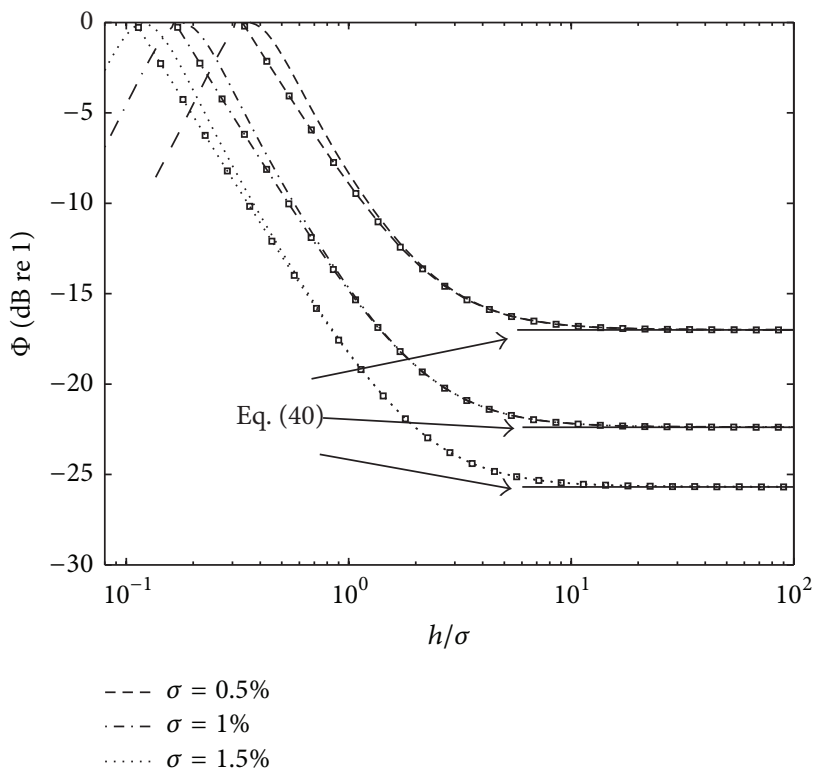

FIGURE 6: The effect of perforation for plates with medium size holes; $d_{o}=2 \mathrm{~mm}$ (marked line: approximated $\Phi$ at mass controlled region from (39)).

For the solid plate, the TL can be obtained by setting the hole impedance in (35) to a very large value, $h \rightarrow \infty$, or perforation ratio equals zero, $\sigma=0$, which gives

$$
\mathrm{TL} \approx 20 \log _{10}(\omega m)-58 \text {. }
$$

Note that (34), (35), and (36) are based on the assumption that the mechanical impedance is much larger than the impedance of the air, $j \omega m \gg z_{f}$ which might not be valid for a light material, for example, a thin plastic sheet.

3.3. The Effect of Perforation. It is also of interest to determine the "loss" of the transmission loss due to perforation which quantifies the $\mathrm{dB}$ reduction obtained by installing a perforated partition instead of a solid panel. This is defined as the ratio of the transmitted power after to that before the perforation. In terms of transmission coefficient $\tau$ and TL, the effect of perforation is therefore expressed as

$$
\Phi=10 \log _{10}\left(\frac{\tau_{s}}{\tau_{p}}\right)=\mathrm{TL}_{p}-\mathrm{TL}_{s}
$$

where subscripts $s$ and $p$ refer to solid plate and perforated plate, respectively.

Figure 4 presents the effect of perforation from results in Figure 3. Figure 4(a) shows that for the plates with micro size holes below the natural frequency $(40 \mathrm{~Hz})$, there is no effect of perforation; that is, $\Phi \approx 0$ for $d_{o}=1 \mathrm{~mm}$ (see also Figure 4(b)). The $\Phi$ can be seen to decrease by increasing the hole diameter. Above the natural frequency in the "normal incidence mass law" region, $\Phi$ decreases by $20 \mathrm{~dB} / \mathrm{decade}$. It then converges towards a constant level at very high frequency (in this case above $10 \mathrm{kHz}$ ) when the imaginary part of the hole impedance is dominant. At this 


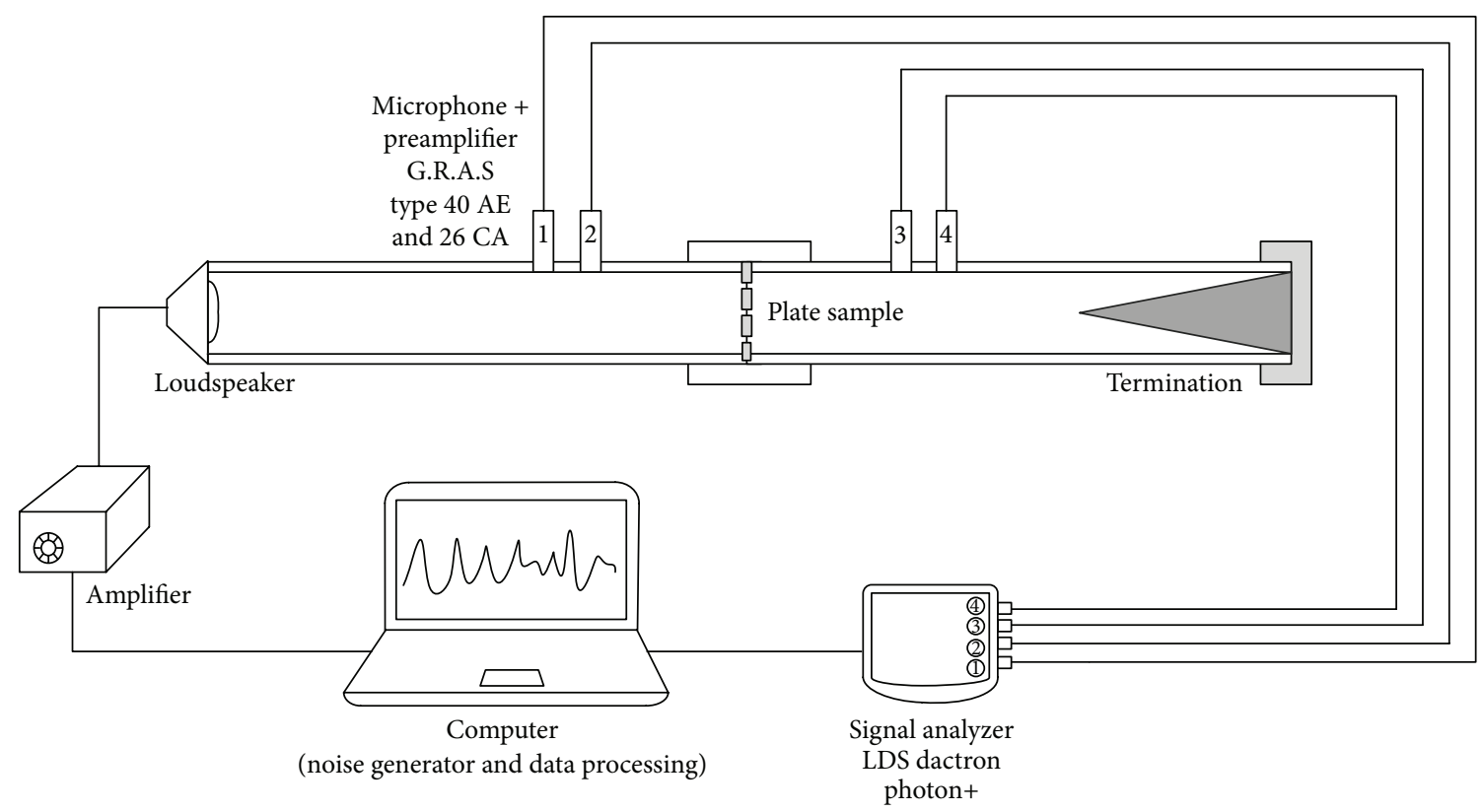

FIGURE 7: Diagram of the experimental setup.

mass controlled region, substituting (34) and (36) into (37) the effect of perforation can be approximated by

$$
\Phi=10 \log _{10}\left(\frac{z_{h, I}^{2}+\left[z_{h, R}+2 z_{f}\right]^{2}}{z_{h, R}^{2}+\left[\left|z_{h, I}\right|-\omega m\right]^{2}}\right) .
$$

The effect of perforation for different perforation ratios from Figure 4(b) is compared with the results from (38) as shown in Figure 5 with good agreement.

For the results of plates with macro size hole as shown in Figures 4(c) and 4(d), below the natural frequency, $\Phi$ has the same level and trend as the STL of the solid plate which is controlled by the stiffness of the mounting. At the mass controlled region, same as for the micro holes at very high frequency, $\Phi$ also decreases by $20 \mathrm{~dB} /$ decade before converging to a constant level depending on the hole diameter and perforation ratio. Again, substituting (35) and (36) into (37) yields

$$
\Phi=10 \log _{10}\left(1+\frac{4 \sigma^{2}}{h^{2}}\right)-20 \log _{10}\left(1+\frac{\omega m \sigma}{h z_{f}}\right),
$$

where on the right hand side, the first term is independent of frequency (as the nondimensional acoustic reactance at the denumerator is proportional to frequency, $h \propto \omega)$ and therefore determines the corresponding constant value (in this case above $1 \mathrm{kHz}$ ). The second term controls the $20 \mathrm{~dB} /$ decade slope right after the undamped natural frequency where this term reduces as the frequency increases. This second term vanishes when $4 \sigma^{2} / h^{2} \ll 1$ or $h / \sigma \gg 2$ and hence gives

$$
\Phi=-20 \log _{10}\left(1+\frac{\omega m \sigma}{h z_{f}}\right)
$$

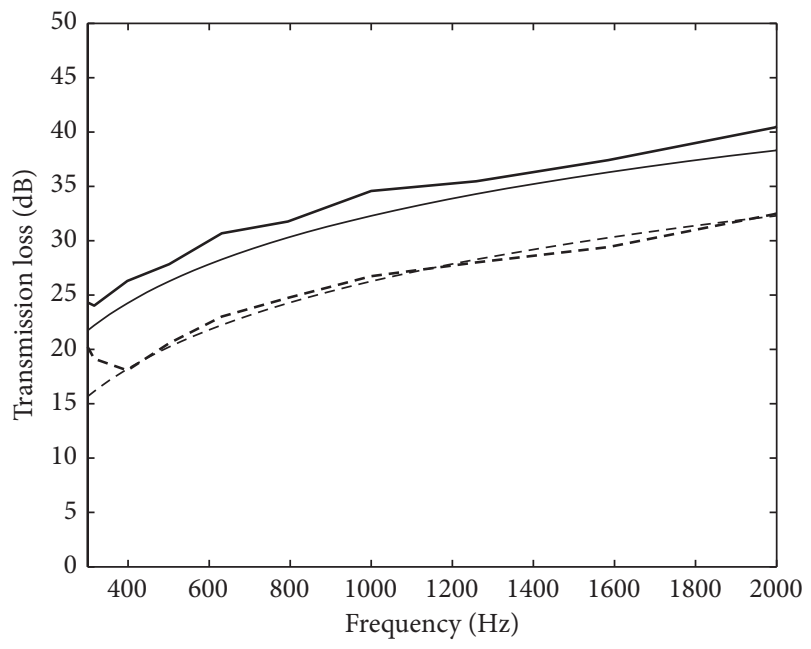

FIGURE 8: Validation of the impedance tube system using the transmission loss data of solid panels (aluminium plate, $--t=1 \mathrm{~mm}$ and $-t=2 \mathrm{~mm}$; measured (thick line) and theory (thin line).

Figure 6 plots the results as in Figure 4(d) and those from (39) and (40). It can be seen that (40) is valid at frequency equivalent to roughly $h / \sigma>10$. Comparison with the results using the complete formulae as in Figure 4(d) gives good agreement except small discrepancy at the $20 \mathrm{~dB} / \mathrm{dec}$ ade slope by $1 \mathrm{~dB}$ particularly for $\sigma=0.5 \%$.

\section{Experimental Validation}

An experimental work has been conducted to measure the sound transmission loss of perforated plates with various hole diameters and perforation ratios. Figure 7 shows 


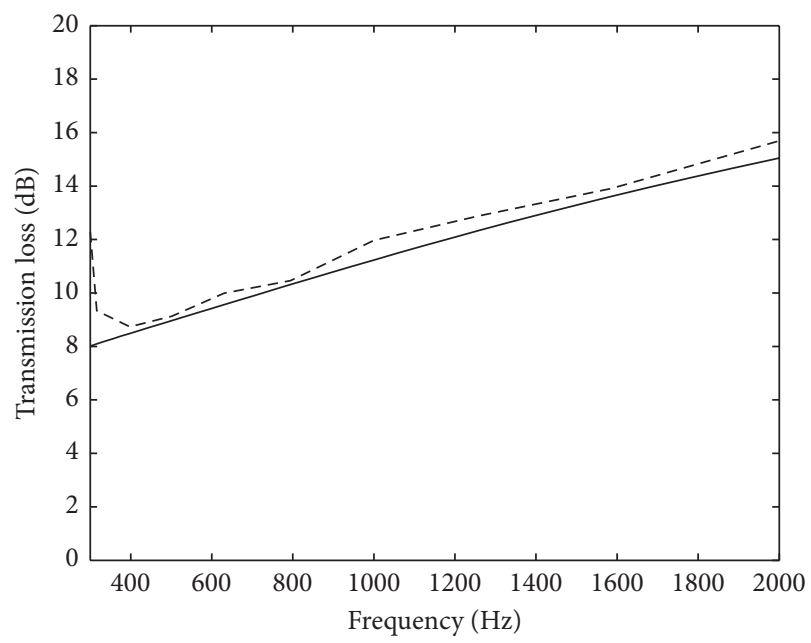

(a)

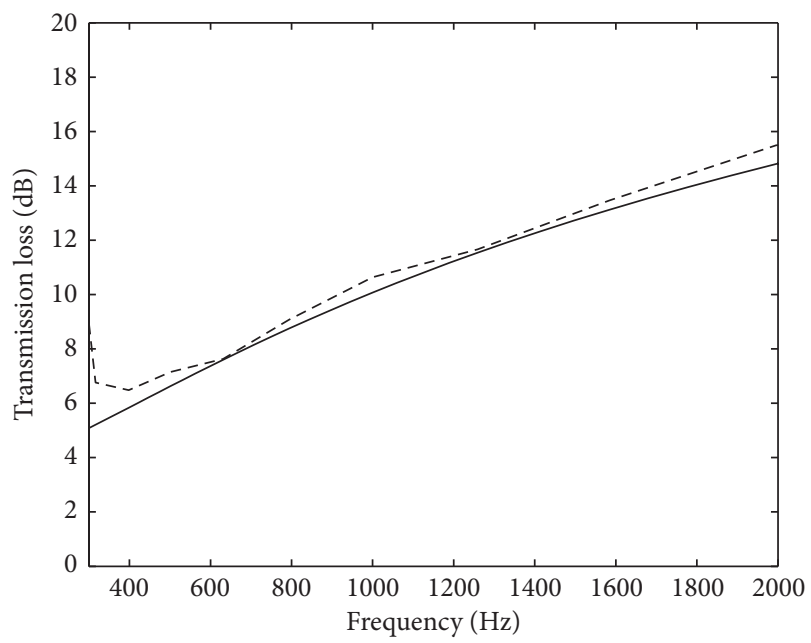

(c)
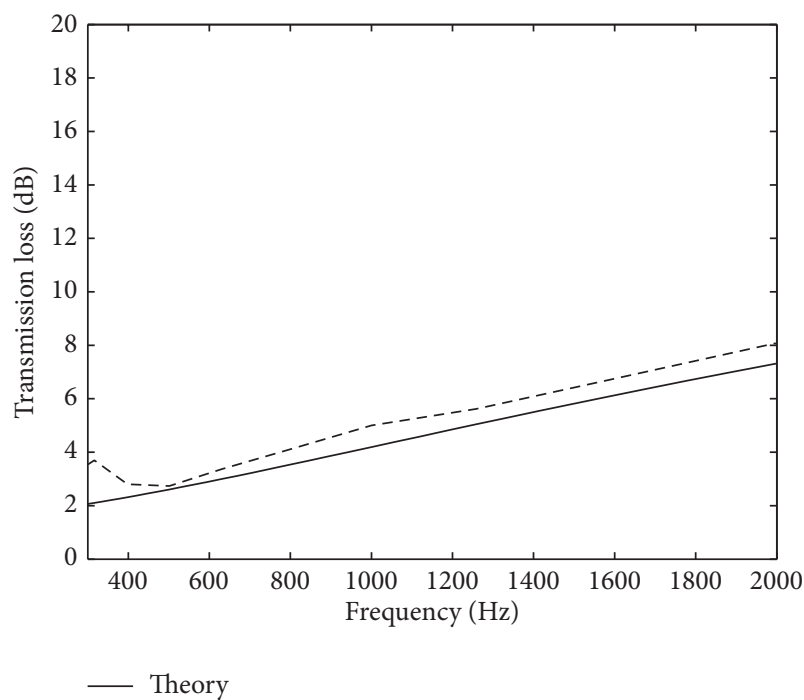

- - - Experiment

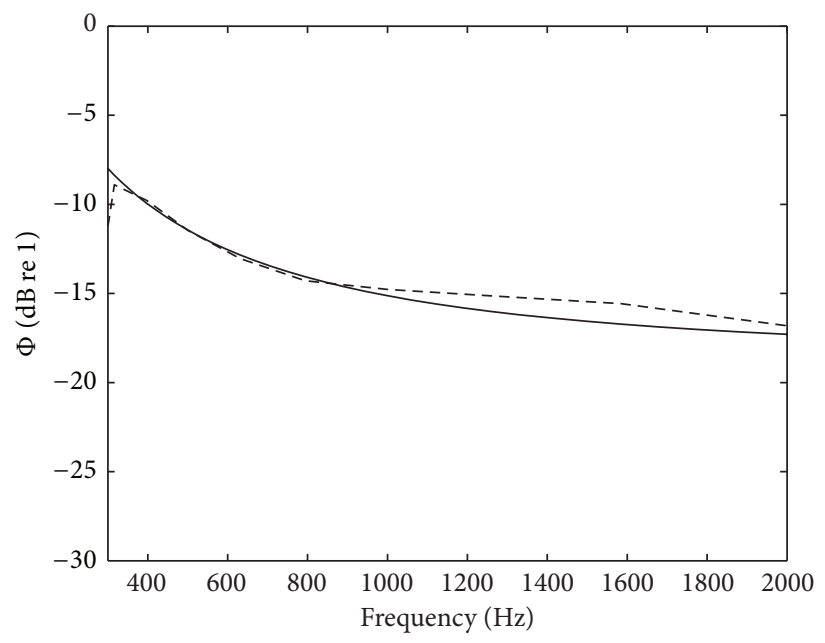

(b)

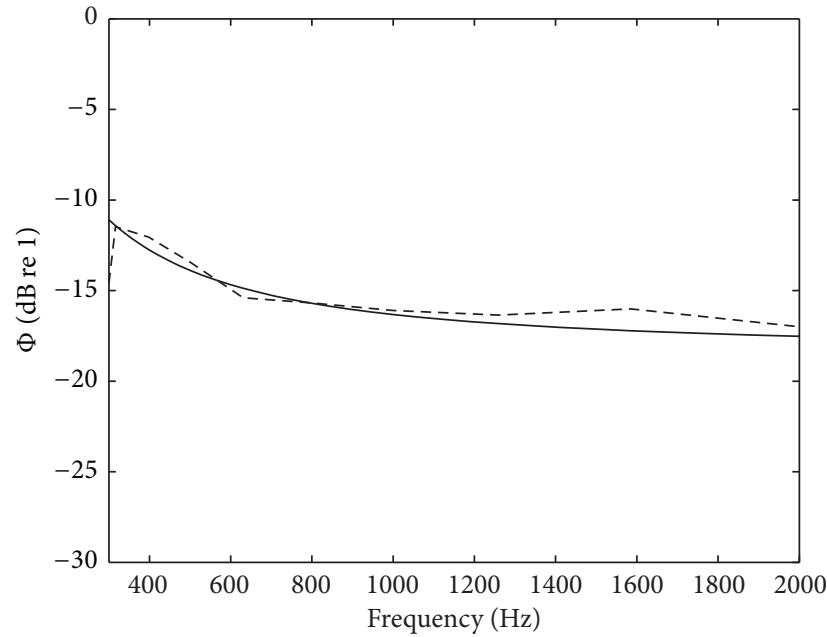

(d)

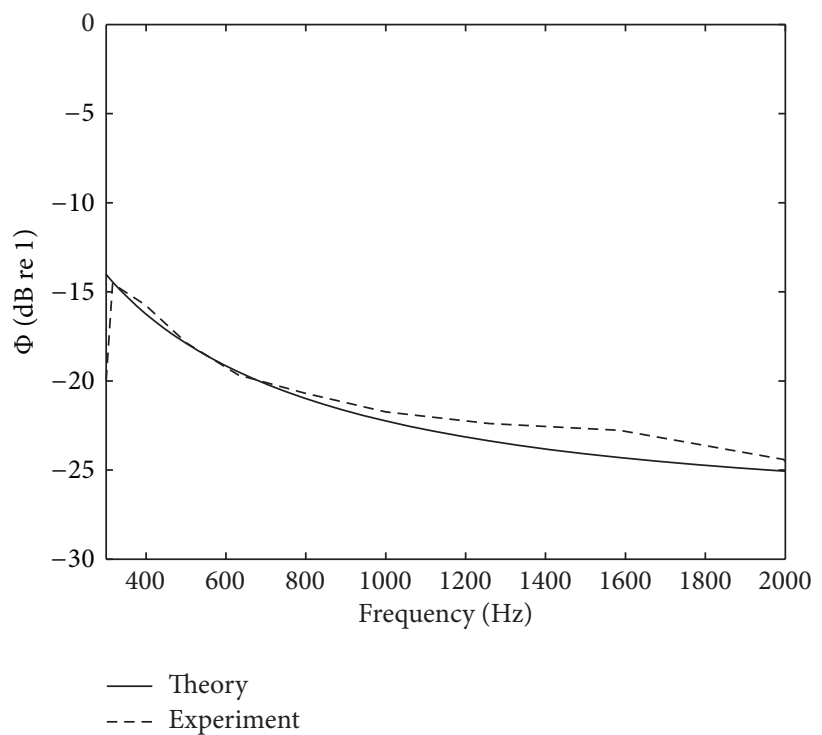

(f)

Figure 9: Measured sound transmission loss (left column) and effect of perforation (right column) of plates with micro size holes: (a) $d_{o}=$ $0.3 \mathrm{~mm}, \sigma=0.5 \%$, (b) $d_{o}=0.5 \mathrm{~mm}, \sigma=0.5 \%$, and (c) $d_{o}=0.5 \mathrm{~mm}, \sigma=1.5 \%$. 


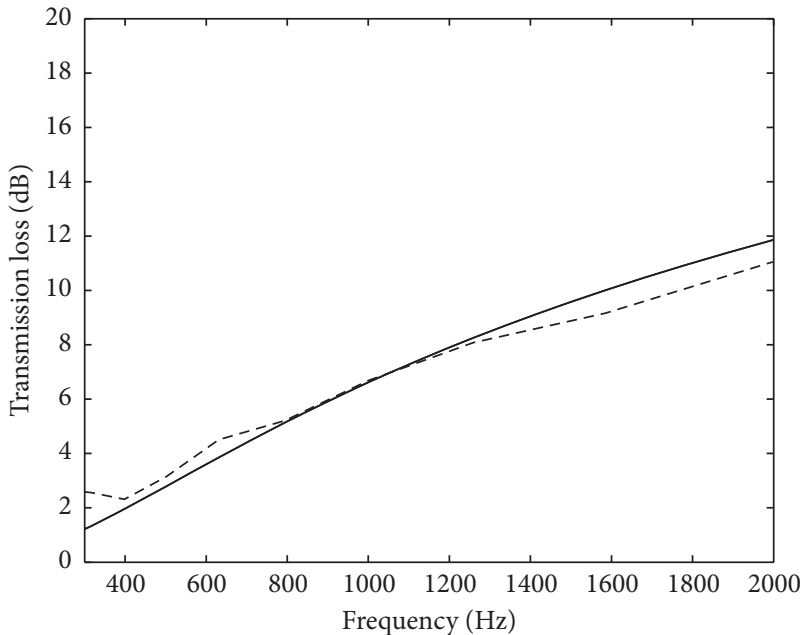

(a)

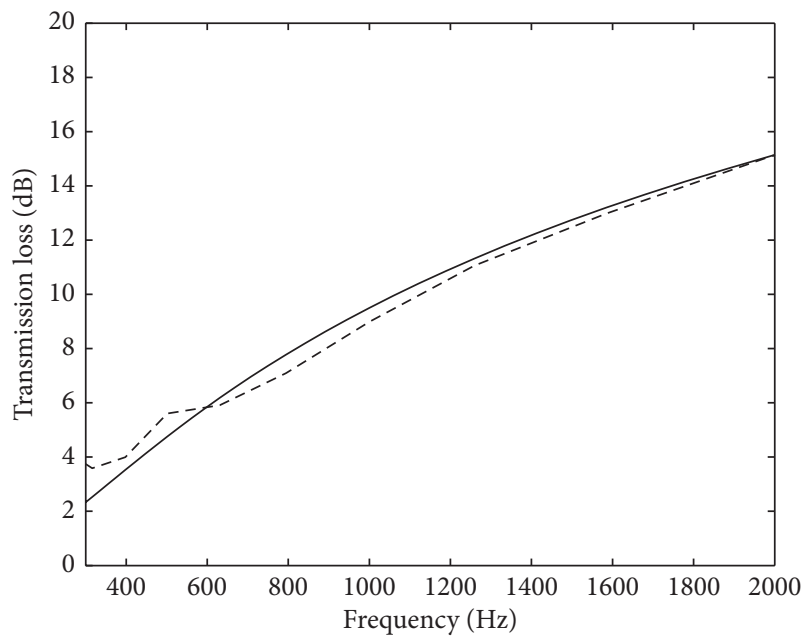

(c)

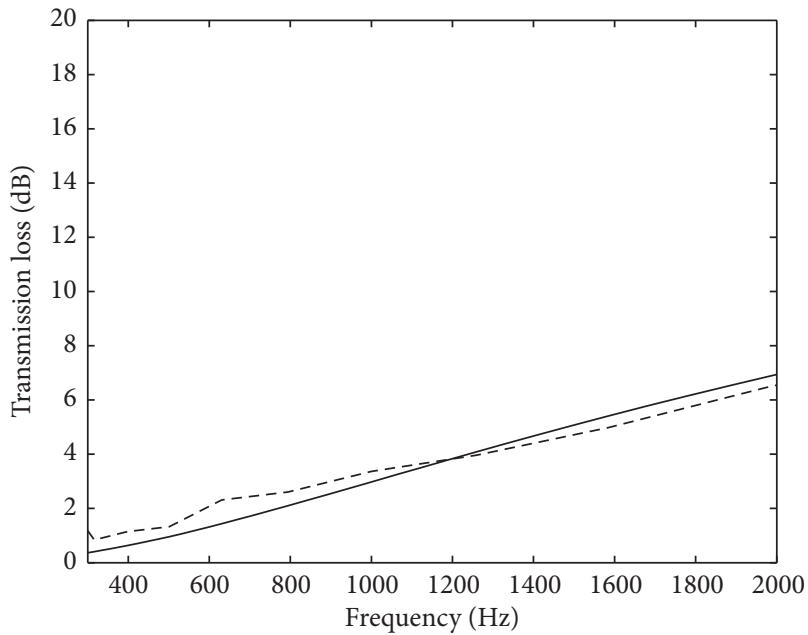

- Theory

--- Experiment

(e)

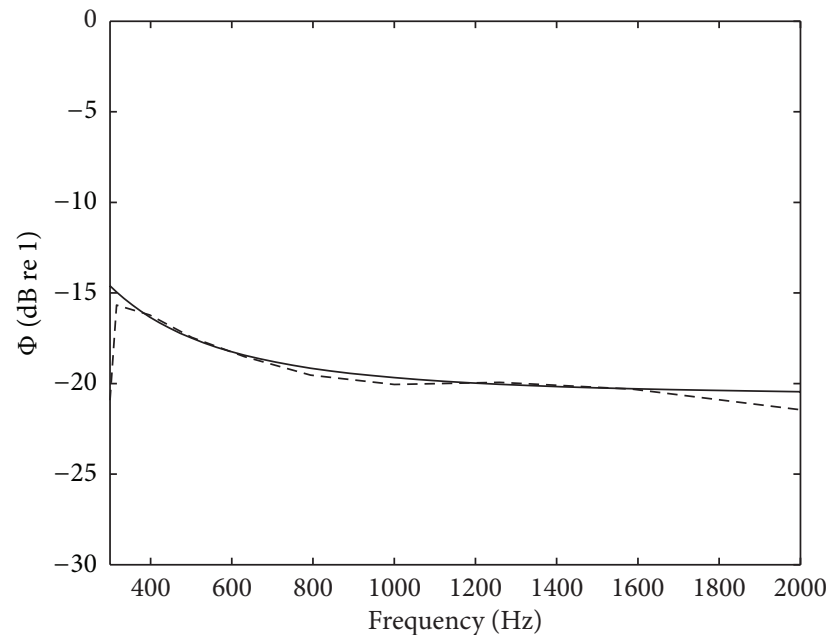

(b)

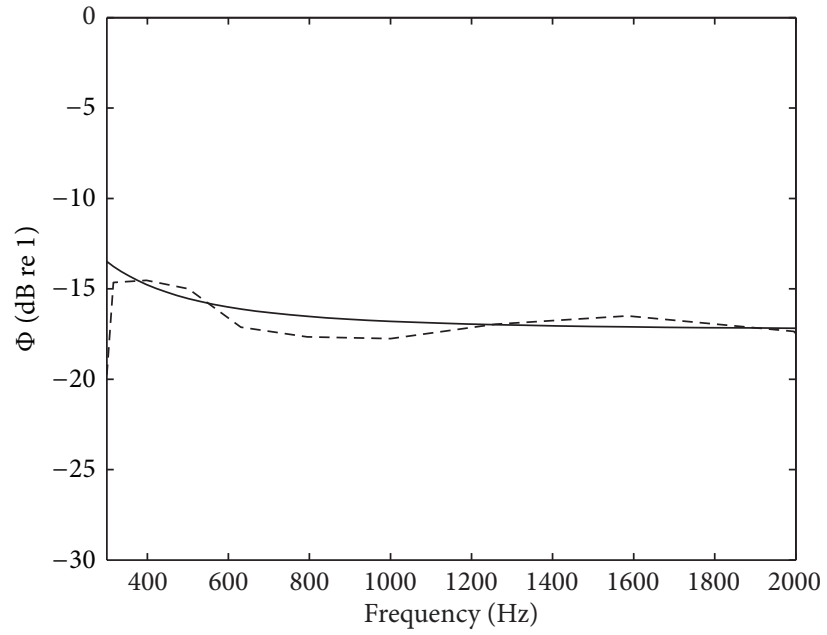

(d)

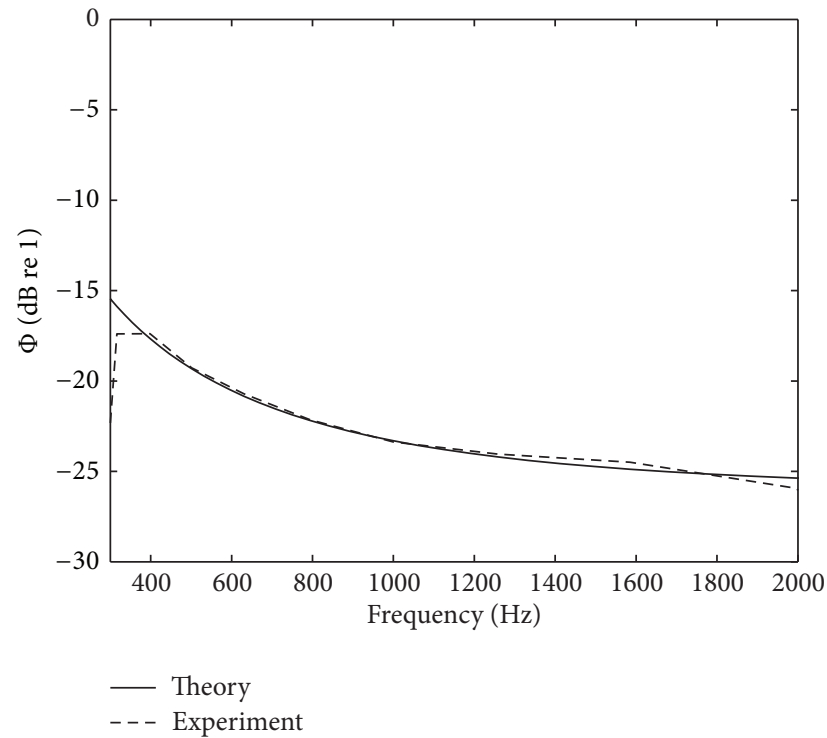

(f)

FIGURE 10: Measured sound transmission loss (left column) and effect of perforation (right column) of plates with macro size holes: (a) $d_{o}=1.5 \mathrm{~mm}, \sigma=1 \%$, (b) $d_{o}=3 \mathrm{~mm}, \sigma=1 \%$, and (c) $d_{o}=1.5 \mathrm{~mm}, \sigma=2 \%$. 
the experimental setup using an impedance tube to measure the STL for normal incidence of acoustic loading. The impedance tube uses $10 \mathrm{~mm}$ thick aluminium tube having inner diameter of $50 \mathrm{~mm}$ and a total length of $960 \mathrm{~mm}$ for downstream tube (between sound source and sample) and upstream tube (between sample and termination). Four 1/2inch G.R.A.S acoustic microphones were used with Pro Photon 6.34 signal analyzer to record the sound pressure inside the tube. The recorded signals were then processed using MATLAB to obtain the transmission coefficient.

The plate samples are made from $1 \mathrm{~mm}$ thick aluminum where the hole diameter is varied with $0.3 \mathrm{~mm}$ and $0.5 \mathrm{~mm}$ micro size holes and by $1.5 \mathrm{~mm}$ and $3 \mathrm{~mm}$ macro size holes with perforation ratios of $0.5 \%$ and $1 \%$. The sample is attached at the inner tube using elastic paper tape at the plate edge. The light tape was ensured to only provide negligible additional mass on the plate and was applied uniformly around the plate edges which can act as the spring element as in Figure 1.

The experiment employed the signal processing technique proposed by Salissou and Panneton [16]. This method requires two different loads for termination. A conical block from glass wool with length $12 \mathrm{~cm}$ was used as one of the loads to approximately simulate the anechoic termination. The other end load was a cylinder shape absorbent also made from $3 \mathrm{~cm}$ thick of glass wool. Figure 8 presents the measurement results (plotted in one-third octave bands with linear frequency) of transmission loss for solid plates which can be seen to give good agreement with theory.

Figure 9 shows the measured results from plate samples with micro holes compared with the simulation results from (34). The effect of perforation is also plotted to validate the measured data with (38). Based on the diameter of the tube, the result is only valid from around $300 \mathrm{~Hz}$ [16]. Good agreement with small discrepancy of less than $1 \mathrm{~dB}$ can be seen for the STL except below $500 \mathrm{~Hz}$ where the measured results overestimate the theory. This might be due to the conical termination where it is difficult to achieve anechoic condition at low frequencies. For the effect of perforation $\Theta$, small discrepancy above $1 \mathrm{kHz}$ is shown as the results of disagreement from both the STL of perforated and solid plates with the theory. Figure 10 presents the results from the samples with macro size holes which also shows good agreement above $500 \mathrm{~Hz}$. The measurement therefore validates that, for plates with micro size holes, by retaining the perforation ratio, the TL can be increased by reducing the hole diameter (see Figures 9(a), 9(b), 9(c), and 9(d)) and for those with macro size holes is by increasing the hole diameter (see Figures 10(a), 10(b), 10(c), and 10(d)).

\section{Conclusions}

Analytical models to determine sound transmission loss for perforated plates having submillimeter and millimeter size holes have been reported. It is found that for the former where the resistive part is dominant for almost entire of the frequency range, the STL reduces as the diameter increases with fixed perforation ratio. On the contrary, for the latter where the reactive part is much greater than the resistive part, the STL increases as the diameter is increased. Simple analytical models of STL for mass controlled region are also proposed. The analytical results have also been validated with measured data from impedance tube with good agreement.

\section{Conflict of Interests}

The authors declare that there is no conflict of interests regarding the publication of this paper.

\section{Acknowledgment}

The authors gratefully acknowledge the financial support provided for this research by the Ministry of Higher Education Malaysia (MoHE) under Fundamental Research Grant Scheme no. FRGS/2010/FKM/TK02/3-F0078.

\section{References}

[1] D. Y. Maa, "Theory and design of microperforated panel sound absorbing constructions," Scientia Sinica, no. 18, pp. 55-71, 1975 (Chinese).

[2] Y. Y. Lee, E. W. M. Lee, and C. F. Ng, "Sound absorption of a finite flexible micro-perforated panel backed by an air cavity," Journal of Sound and Vibration, vol. 287, no. 1-2, pp. 227-243, 2005.

[3] J. Pfretzschner, P. Cobo, F. Simón, M. Cuesta, and A. Fernández, "Microperforated insertion units: an alternative strategy to design microperforated panels," Applied Acoustics, vol. 67, no. 1, pp. 62-73, 2006.

[4] H. V. Fuchs and X. Zha, "Micro-perforated structures as sound absorbers-a review and outlook," Acta Acustica united with Acustica, vol. 92, no. 1, pp. 139-146, 2006.

[5] K. Sakagami, M. Morimoto, and W. Koike, "A numerical study of double-leaf microperforated panel absorbers," Applied Acoustics, vol. 67, no. 7, pp. 609-619, 2006.

[6] M. Toyoda, M. Tanaka, and D. Takahashi, "Reduction of acoustic radiation by perforated board and honeycomb layer systems," Applied Acoustics, vol. 68, no. 1, pp. 71-85, 2007.

[7] A. Putra and D. J. Thompson, "Sound radiation from perforated plates," Journal of Sound and Vibration, vol. 329, no. 20, pp. 4227-4250, 2010.

[8] K.-T. Chen, "Study on the acoustic transmission loss of a rigid perforated screen," Applied Acoustics, vol. 47, no. 4, pp. 303-318, 1996.

[9] D. Takahashi and M. Tanaka, "Flexural vibration of perforated plates and porous elastic materials under acoustic loading," Journal of the Acoustical Society of America, vol. 112, no. 4, pp. 1456-1464, 2002.

[10] F. Asdrubali and G. Pispola, "Properties of transparent soundabsorbing panels for use in noise barriers," Journal of the Acoustical Society of America, vol. 121, no. 1, pp. 214-221, 2007.

[11] M. Toyoda and D. Takahashi, "Sound transmission through a microperforated-panel structure with subdivided air cavities," Journal of the Acoustical Society of America, vol. 124, no. 6, pp. 3594-3603, 2009.

[12] R. L. Mu, M. Toyoda, and D. Takahashi, "Sound insulation characteristics of multi-layer structures with a microperforated panel," Applied Acoustics, vol. 72, no. 11, pp. 849-855, 2011. 
[13] A. Putra, A. Y. Ismail, R. Ramlan, R. Md. Ayob, and M. S. Py, "Normal incidence of sound transmission loss of a double-leaf partition inserted with a microperforated panel," Advances in Acoustics and Vibration, vol. 2013, Article ID 216493, 8 pages, 2013.

[14] A. D. Pierce, Acoustics, Acoustical Society of America, 1989.

[15] F. J. Fahy and P. Gardonio, Sound and Structural Vibration: Radiation, Transmission and Response, Academic Press, London, UK, 2nd edition, 2006.

[16] Y. Salissou and R. Panneton, "A general wave decomposition formula for the measurement of normal incidence sound transmission loss in impedance tube," Journal of the Acoustical Society of America, vol. 125, no. 4, pp. 2083-2090, 2009. 

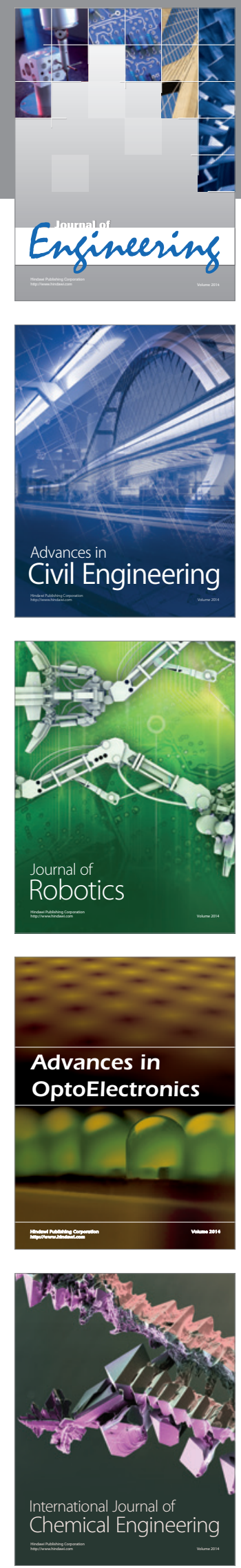

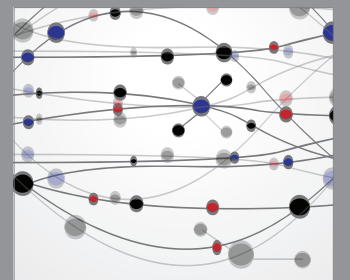

The Scientific World Journal
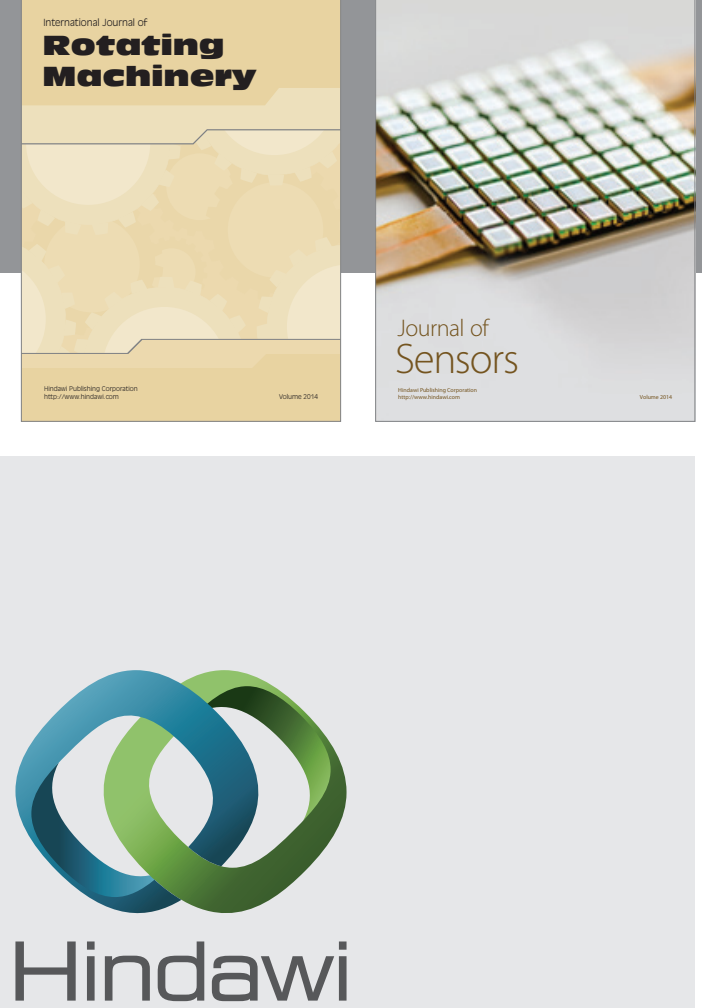

Submit your manuscripts at http://www.hindawi.com
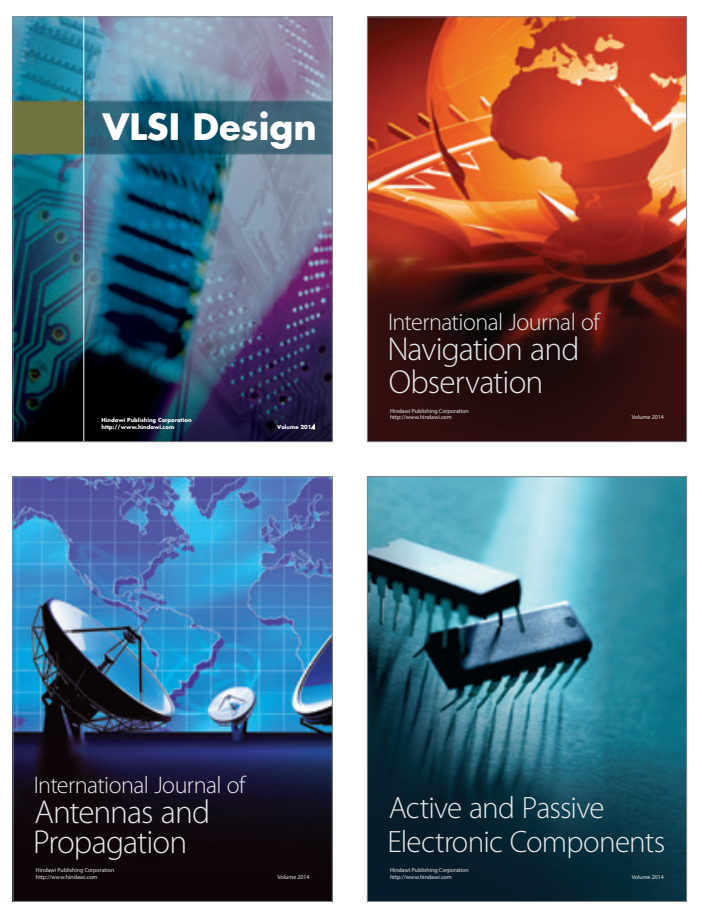
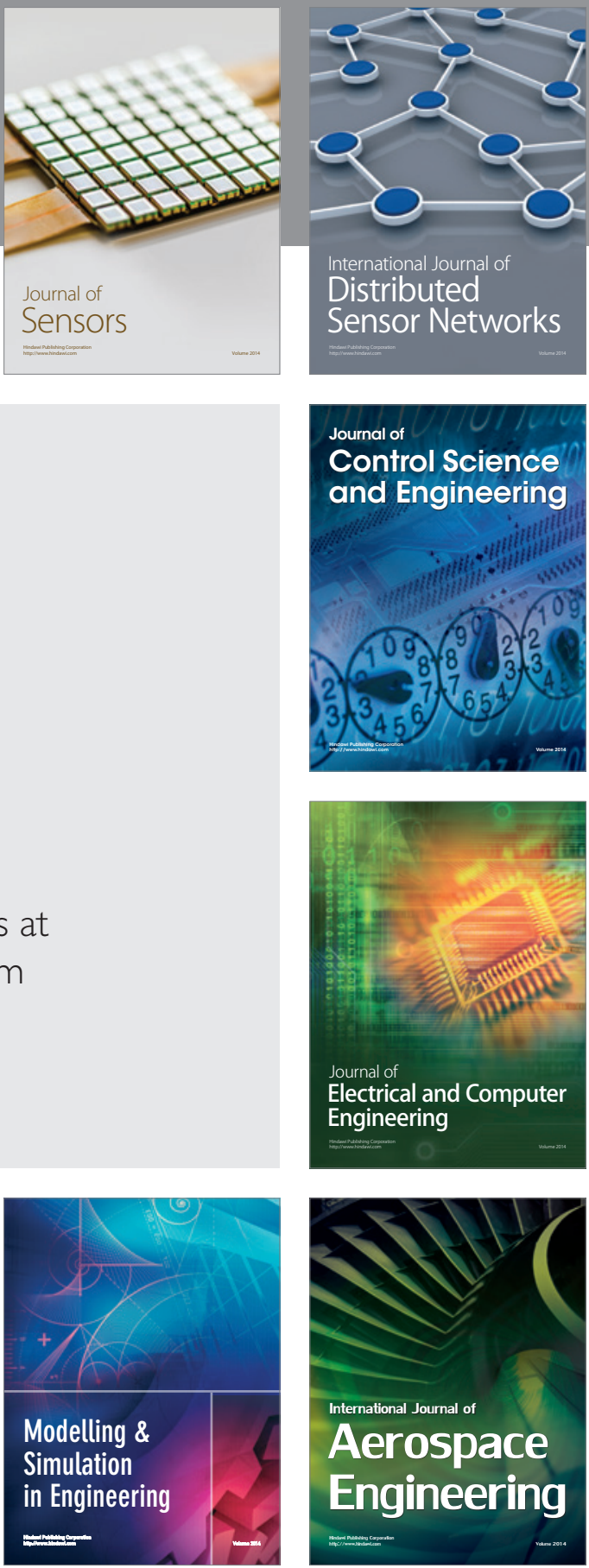

Journal of

Control Science

and Engineering
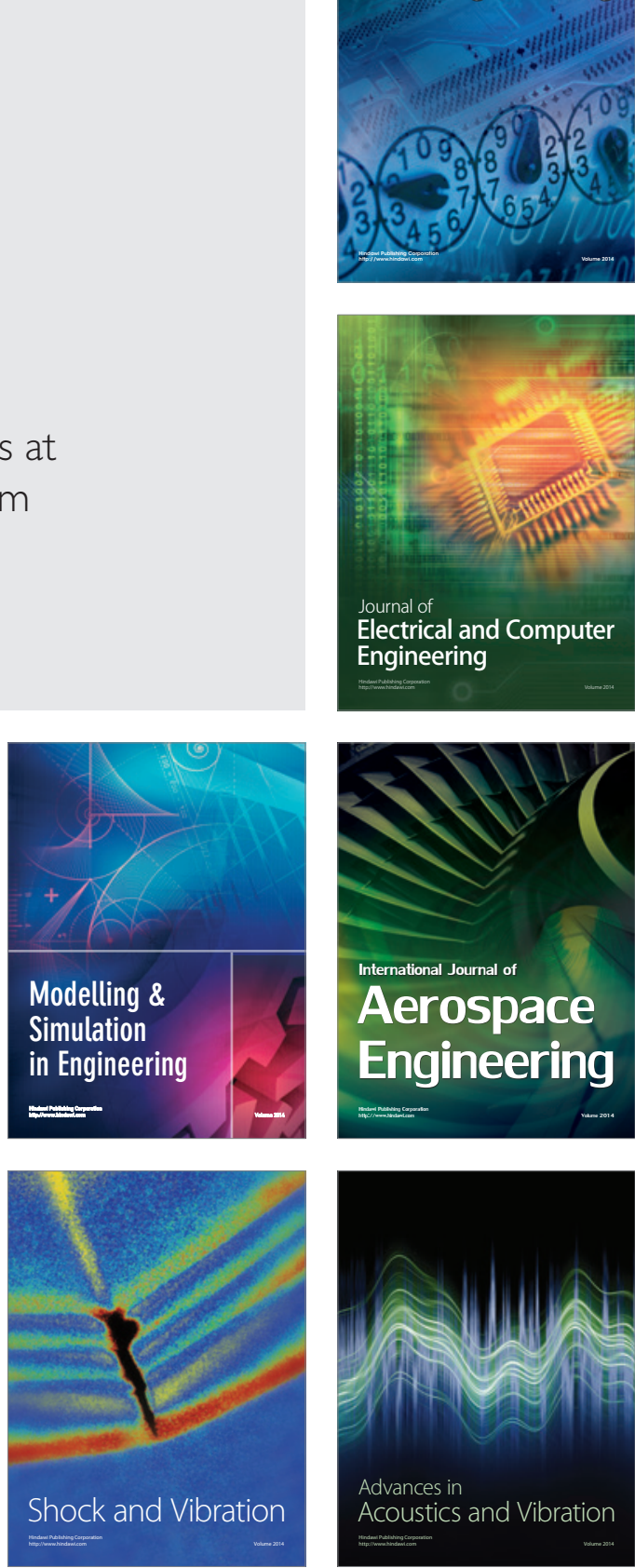